\title{
Simultaneous Quantification of Cortisol and Cortisone in Serums and Saliva from Depressive Patients by Supported Liquid Extraction Coupled to HPLC-MS/MS
}

\author{
Binbin Chen ${ }^{1}$, Haiyan Lyu ${ }^{1}$, Xiangzhen $\mathrm{Xu}^{1}$ and Chen Wang ${ }^{2 *}$ \\ 'Xiamen Medical College affiliated Xiamen Xianyue Hospital, 387 Xianyue Road, Xiamen 361012, China \\ ${ }^{2}$ Xiamen Medical College, 1999 Guankou Middle Road, Xiamen 361023, China
}

Received: 30 Oct 2019; accepted: 13 Dec 2019

\begin{abstract}
Cortisol and cortisone are 2 important glucocorticoids produced in the human hypothalamus-pituitary-adrenal (HPA) axis that respond to stress. An analytical method to determinate cortisol and cortisone in serum and saliva using high-performance liquid chromatography-tandem mass spectrometry following a supported liquid extraction (SLE) was developed. Serum and saliva samples of $0.2 \mathrm{~mL}$ were extracted by SLE three times using $0.4 \mathrm{~mL}$ of methyl tert-butyl ether each time. The chromatographic separation was obtained on an Agilent Poroshell column using a $0.01 \%$ formic acid buffer and acetonitrile $(60: 40, v / v)$ as the solvent with a flow rate of $0.3 \mathrm{~mL} / \mathrm{min}$. Optimized quantitative mass transitions for cortisol, cortisone, and cortisone d-4 were 363.2/121.0 (m/z), 361.2/163.1 $(\mathrm{m} / \mathrm{z})$, and $367.1 / 270.7(\mathrm{~m} / \mathrm{z})$, respectively. The method validation was achieved according to regulatory guidance. The lower limit of quantification (LLOQ) in serum were $2 \mathrm{ng} / \mathrm{mL}$ for cortisol and $1 \mathrm{ng} / \mathrm{mL}$ for cortisone, and the LLOQ in saliva were $0.1 \mathrm{ng} / \mathrm{mL}$ for cortisol and $0.2 \mathrm{ng} / \mathrm{mL}$ for cortisone. The developed method showed convenient and efficient extraction, a lower LLOQ, and a short running time. Modest correlations between serum and saliva cortisol and cortisone concentrations were found. The method was successfully applied in assessing the HPA condition of patients with depressive disorders.
\end{abstract}

Keywords: cortisol, cortisone, LC-MS/MS, supported liquid extraction

\section{Introduction}

Cortisol and cortisone are 2 of the main glucocorticoid hormones secreted by the adrenal gland; they play an important role in the immune system, stress-responsive system, and cardiovascular system [1]. In a normal state, the body can balance the secretion of cortisol and cortisone. However, in some cases the amount of cortisol and cortisone can change, and these changes may affect health, leading to the occurrence and development of diseases. The function of the hypothalamuspituitary-adrenal (HPA) axis is widely described as a stress biomarker [2]. Furthermore, high levels of cortisol and cortisone were found in individuals who have attempted suicide [3-5] and individuals with depressive disorders [6, 7] and psychiatric diseases $[8,9]$. The determination of cortisol and cortisone helps to assess HPA-axis function and the stress state of depressive patients.

Cortisol and cortisone are present in serum and saliva. Serum is the most common biological sample and is suitable for routine examination. Saliva collection is a noninvasive, stressfree measurement, and salivary cortisol and cortisone show promising correlation to serum cortisol and cortisone [10]. Salivary cortisol measurement offers a reliable alternative to measuring free cortisol in serum. Due to their importance in diagnosis and in clinical settings, there are many analytical methods for the measurement of cortisol and cortisone in the literature. During the past few years, liquid chromatographymass spectrometry (LC-MS) or liquid chromatography-

\footnotetext{
*Author for correspondence: Xiamen Medical College, 1999 Guankou Middle Road, Xiamen, Fujian Province, China. Tel/Fax: +86 15959290351; fjxmstorm@hotmail.com.
}

tandem mass spectrometry (LC-MS/MS) has become a mature technique with many applications to various biological matrixes in cortisol and cortisone analyzing fields [11-18]. However, most of the methods suffered from poor high-throughput utilization and extraction efficiency.

The aim of this work is to establish a validated, rapid, and reliable method for determination of cortisol and cortisone commonly used in clinical laboratories. The supported liquid extraction (SLE) method was used for extraction and high-performance liquid chromatography-tandem mass spectrometry (HPLC-MS/MS) was optimized for analyzing the concentration of cortisol and cortisone. The method was successfully applied for the quantification of these 2 steroid hormones in serum and saliva.

\section{Experimental}

Chemicals and Standards. Cortisol and cortisone (purity $\geq$ 98\%) were purchased from the National Institutes for Food and Drug Control (Beijing, China), and cortisol-d4 was purchased from Toronto Research Chemicals (Toronto, Canada). Structures of all the compounds are given in Figure 1. All the HPLC-grade solvents, including methanol, acetonitrile, and methyl tert-butyl ether (MTBE), were purchased from the Sigma-Aldrich Company (St. Louis, MO, USA). Certified charcoal stripped fetal bovine serum (cortisoland cortisone-free calf serum) was obtained from Biological Industries (Haifa, Israel). Artificial saliva was purchased from Beijing Solarbio Life Sciences (Beijing, China). Triple distilled deionized water was obtained from Millipore Water Purification Systems (Molsheim, France).

This is an open-access article distributed under the terms of the Creative Commons Attribution-NonCommercial 4.0 International License (https://creativecommons.org/licenses/by-nc/4.0/), which permits unrestricted use, distribution, and reproduction in any medium for non-commercial purposes, provided the original author and source are credited, a link to the CC License is provided, and changes - if any - are indicated. 
<smiles>C[C@]12CC(O)C3C(CCC4=CC(=O)CC[C@@]43C)C1CC[C@]2(O)C(=O)CO</smiles>

(a) cortisol<smiles>C[C@]12CCC(=O)C=C1CCC1C2C(=O)C[C@]2(C)C1CC[C@@]2(O)C(=O)CO</smiles>

(b) cortisone

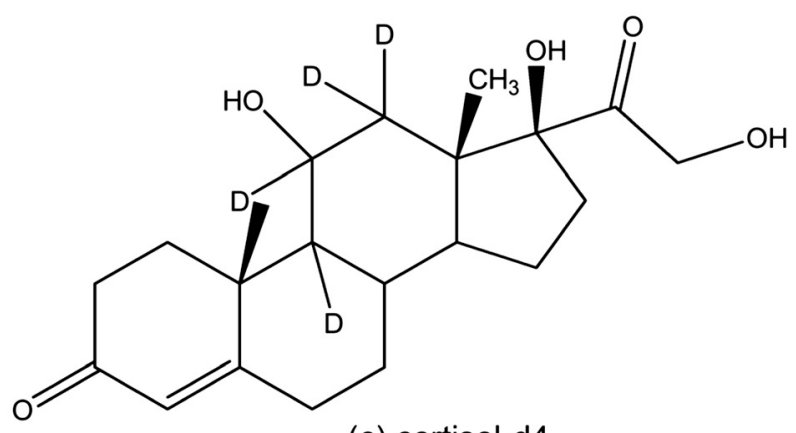

(c) cortisol-d4

Figure 1. The chemical structure of (a) cortisol, (b) cortisone, and (c) cortisol-d4

Stock and Standard Solutions and Quality Control Sample Preparation. Stock solution mixed with cortisol (20 $\mu \mathrm{g} / \mathrm{mL})$ and cortisone $(10 \mu \mathrm{g} / \mathrm{mL})$ was prepared in methanol. The internal standard (IS) solution of cortisol-d4 was prepared in methanol at a concentration of $100 \mathrm{ng} / \mathrm{mL}$. Serum calibrator standard solutions were prepared by diluting the stock solution in methanol. Quality control (QC) solutions of serum were prepared with stock solutions at 4 different levels. All the stock solutions were stored at $4{ }^{\circ} \mathrm{C}$. Serum calibrators were prepared by spiking $10 \mu \mathrm{L}$ of a serum calibrator standard (CS) with $190 \mu \mathrm{L}$ of calf serum to establish the final calibration. The serum cortisol calibrators were $2-200 \mathrm{ng} / \mathrm{mL}$, and the serum cortisone calibrators were $1-100 \mathrm{ng} / \mathrm{mL}$. The saliva calibrator standards solutions were prepared with stock solutions via dilution in methanol. The saliva calibrators were prepared by diluting the saliva calibrator standard solutions in artificial saliva to a final concentration of $0.1-20 \mathrm{ng} / \mathrm{mL}$ for cortisol and $0.2-40 \mathrm{ng} / \mathrm{mL}$ for cortisone.

A pooled matrix of serum or saliva with cortisol and cortisone were prepared from 20 different individuals. QCs of cortisol and cortisone in serum were prepared as follows: the lower limit of quantification (LLOQ) and low QC (LQC) were the calf serums spiked with QC solutions, and the medium QC (MQC) and high QC (HQC) were the pooled serum matrix spiked with MQC and HQC stock solutions. Saliva QCs were prepared using the same method as the serum QCs.

Chromatographic and Mass Spectrometry Conditions. The chromatography system consisted of high-performance liquid chromatography (1260 HPLC system, Agilent Technologies, Waldbronn, Germany) and a mass spectrometry system (6460C, Agilent Technologies, Waldbronn, Germany). The ionization was performed in the positive mode with an electrospray ionization source. The detection was operated on an Agilent $6460 \mathrm{C}$ triple quadrupole mass spectrometer. The chromatographic separation was obtained on an Agilent Poroshell 120, EC-C18 $(2.1 \mathrm{~mm} \times 50 \mathrm{~mm}, 2.7 \mu \mathrm{m})$ column using acetonitrile and a $0.01 \%$ formic acid buffer $(40: 60, v / v)$ as the solvent with a flow rate of $0.3 \mathrm{~mL} / \mathrm{min}$, and the column temperature was $40{ }^{\circ} \mathrm{C}$. The mass spectrometric detection of cortisol, cortisone, and the IS was operated in the multiple reaction monitoring mode. The drying gas flow rate was $11 \mathrm{~L} /$ min, the drying gas temperature was $350{ }^{\circ} \mathrm{C}$, the atomizer pressure was $35 \mathrm{psi}$, and the capillary voltage was $4000 \mathrm{~V}$. High-purity nitrogen was used as the nebulizing gas. Two selected ion transitions were detected as an ion quantifier and an ion qualifier. Cortisol was detected at $m / z 363.3 \rightarrow 121.1$ and $363.3 \rightarrow 105.1$, and cortisone was detected at $\mathrm{m} / \mathrm{z}$ $361.1 \rightarrow 163.1$ and $361.1 \rightarrow 105.1$. The IS of cortisol-d4 was detected at $\mathrm{m} / \mathrm{z} 367.2 \rightarrow 270.7$ and $367.2 \rightarrow 121.0$. Agilent MassHunter qualitative analysis B.07.00 software was used to generate the data obtained from the analysis.

Sample Preparation. Cortisol and cortisone were extracted from the serum or saliva samples using a SLE method with ISOLUTE products (Biotage, Uppsala, Swedish). A $200 \mu \mathrm{L}$ serum/saliva sample was placed in a 96 -well plate, $10 \mu \mathrm{L}$ of the $100 \mathrm{ng} / \mathrm{mL}$ IS working solution was added, and the mixture was vortex-mixed for 15 s. A $200-\mu \mathrm{L}$ mixed sample was added to the SLE kit, and the analyte was eluted with automated Extrahera ${ }^{\mathrm{TM}}$ (Biotage, Uppsala, Swedish) three times using $0.4 \mathrm{~mL}$ MTBE each time. The eluate was collected, evaporated under nitrogen using a TurboVap 96well evaporator (Biotage, Charlotte, NC, USA), and reconstituted in $100 \mu \mathrm{L}$ of the mobile phase. After vortexing for $1 \mathrm{~min}$, the samples were placed in sample bottles, and $2 \mu \mathrm{L}$ of the processed samples were injected into an HPLC system for analysis.

Method Validation. The method was validated by determining its selectivity, sensitivity, linearity, precision, accuracy, matrix effect, stability, carryover, and dilution integrity according to the published US Food and Drug Administration (FDA) guidelines (2018).

Selectivity. For selectivity testing, 6 different practical serum and saliva samples were collected, and 6 different batches of cortisol- and cortisone-free calf serum and artificial saliva were prepared in the same manner used for the practical samples. The standard solutions were also tested for possible chromatogram interference. The selectivity was confirmed by comparison of chromatograms.

Linearity, Limit of Detection, Accuracy, and Precision. The linearity of cortisol and cortisone were determined by plotting the peak area ratio (area of analyte divided by internal standard area) versus 7 levels of the calibration standards. The limit of detection (LOD) in both serum and saliva samples were determined by serial diluting with blank matrix until the achievement of a signal-to-noise ratio $\geq 3$. Six replicates at 4 different QC concentration (including the LLOQ) in 3 consecutive days were analyzed to assess precision and accuracy. Precision of the analytes were calculated as percentage relative standard deviation (\%RSD), and accuracy was calculated as 
the percentage of the tested level to the nominal level. The intra- and inter-day accuracy and precision (\% RSD) of all the analytes were within $\pm 15 \%$ at LQC, MQC, and HQC and within $\pm 20 \%$ at LLOQ.

Extraction Recovery and Matrix Effects. Extraction recovery of the endogenous analyte shows an added complication. Mean extraction recovery and matrix effect were estimated at low, medium, and high levels. The peak areas of cortisol and cortisone for 6 replicate pooled matrices are extracted $(A)$; peak areas of cortisol and cortisone for QC solutions were spiked into the pooled matrix and extracted $(B)$; peak areas of cortisol and cortisone for the pooled matrix are extracted then spiked with QC solutions $(C)$; and peak areas for the neat QC solutions were determined $(D)$, respectively: matrix factor $(\mathrm{MF})=(C-A) /(D) \times 100 \%$; extraction recovery $(\mathrm{ER})=(B-$ $A) /(C-A) \times 100 \%$. The MF coefficient of variation $(\mathrm{CV})$ should be less than $15 \%$.

Stability Studies. The stability of the cortisol and cortisone was tested by evaluating 3 kinds of samples as follows: LQC in blank matrix, HQC in pooled serum or saliva matrix, and practical clinical samples. Stability experiments were carried out in 3 conditions $\left(20{ }^{\circ} \mathrm{C}\right.$ for $24 \mathrm{~h}$, freeze-thaw cycles repeated three times, and 30 days at $-80^{\circ} \mathrm{C}$ ). A freshly prepared linear curve was used for calculating the samples. The concentrations of the stability samples should be between $85 \%$ and $115 \%$ of the baseline concentrations.

Carryover and Dilution Integrity. After determining the upper limit of the calibration samples, a blank sample was immediately injected to determine the peak areas of cortisone, cortisol, and the IS, and the carry-over effects were evaluated. The peak area of cortisol, cortisone, and cortisol-d4 in the blank sample should be less than $20 \%$ of the LLOQ. To cover all the clinical samples, five-times-the-highest calibration curve was diluted with a blank matrix at 10 -fold dilution to evaluate the dilution integrity. The precision and accuracy of the diluted samples should be within $15 \%$ and $\pm 15 \%$ respectively.

Sample Acquisition and Statistical Analysis. Thirty-eight patients with depressive disorders from Xianyue Hospital in Xiamen were enrolled in the study. All patients were diagnosed according to the International Classification of Diseases 10 (ICD-10) diagnostic criteria and composed of 15 males and 23 females aged between 18-60 years. Patients were antidepressant-free for at least 2 weeks, and serums and salivas were collected the morning after admission.

Blood samples were collected in anticoagulant-free Vacutainer (Kangjian, Jiangsu, China) tubes. Salivary samples were collected using commercially available cotton swab Salivette for cortisol determination (AG\&Co, Numbrecht, Germany). The sampling time of the serum and salivary samples were at 6:00 to $6: 30$ in the morning.

Samples were collected and stored in an ultra-low temperature freezer at $-80{ }^{\circ} \mathrm{C}$ and measured within 4 weeks. Informed consents were obtained from all patients. The protocol of the study was approved by the Institutional Review Board of Xiamen Xianyue Hospital Ethics Committee. After obtaining the cortisol and cortisone data, correlations between serum and saliva values were determined using Pearson or Spearman correlation analysis. All tests were carried out using an SPSS 20.0 statistical program. The statistical significance was set at $P<0.05$.

\section{Results and Discussion}

HPLC-MS/MS Optimization. Chromatographic conditions including column, mobile phase composition, and flow rates were optimized to achieve a better peak shape and a shorter running time. Reversed phase columns with different particle sizes and lengths, like Agilent Poroshell and Agilent Zorbax, were assessed for the best peak shape and sensitivity. Both columns obtained a symmetrical peak shape; however, Agilent Poroshell had a lower column pressure. Various mixtures of solvents were made by combining organic phase solvents such as methanol and acetonitrile with aqueous phase buffers of different concentrations, such as formic acid, acetic acid and ammonium acetate. The combination of organic and aqueous phase solvents were tried at different compositions, as well as different flow rates. The addition of $0.1 \%$ formic acid in the aqueous phase solvents showed remarkable improvement in the peak shape and sensitivity of cortisone, cortisol, and cortisol-d4. Thus, Agilent Poroshell EC-C18 $(50 \mathrm{~mm} \times$ $2.1 \mathrm{~mm}, 2.7 \mu \mathrm{m})$ column combined with mobile phases of acetonitrile $-0.01 \%$ formic acid in water $(60: 40, v / v)$ at a flow rate of $0.3 \mathrm{~mL} / \mathrm{min}$ was chosen for the analysis. The best way to reduce interferences of endogenous substances is to use the internal standard. Cortisol-d4 has similar structure to cortisol and has a similar chromatographic behavior to cortisol and cortisone. The chromatographic run time was $2.0 \mathrm{~min}$, which meets the requirements of high-throughput bioanalysis.

Sample Preparation. Among the techniques for biological sample preparation, liquid-liquid extraction (LLE), solidphase extraction (SPE), and protein precipitation (PPT) are widely used. SLE is a fast and clean extraction method which can provide an ideal supporting surface for liquid-liquid distribution and produces no emulsions which can replace most traditional LLE methods. Porous diatom used in SLE as the liquid-liquid distribution carrier has the characteristics of high specific surface area and chemical inertness. The target compounds can be extracted from the aqueous phase by loading and elution. The SLE method has higher recoveries, a lower limit of detection compared to SPE, and LLE. Many SLE methods are reported as being applied in various areas of research [19-21]. When PPT was used to extract analytes from serum and saliva samples, the LLOQ of the serum and saliva were not achieved. When the SPE method was used, it showed unsatisfactory LLOQ in the saliva sample. SLE was therefore chosen for sample preparation. In the optimization procedure, a number of solvents such as dichloromethane methylene chloride (DCM), tert-butyl methyl ether ethyl (TBME), ethyl acetate, and hexane were chosen for elution. Among all the organic solvents studied, TBME gives the maximum recoveries for the analytes and takes a shorter time to evaporate. The developed SLE extraction method is suitable for clinical high-throughput detection.

\section{Method Validation}

Selectivity. The selectivity was assessed by comparing the $\mathrm{MS} / \mathrm{MS}$ responses at the retention times of cortisone, cortisol, and the IS. The SLE extraction and LC-MS/MS methodology provided selectivity and specificity for cortisone, cortisol, and the IS (Figure 2).

Linearity, Accuracy, and Precision. The methods were linear over the concentration ranges of $2-200 \mathrm{ng} / \mathrm{mL}$ for cortisol and $1-100 \mathrm{ng} / \mathrm{mL}$ for cortisone in serum. Because of the different concentrations between saliva and serum, the linearity of cortisol in saliva was in the range of $0.1-20 \mathrm{ng} / \mathrm{mL}$ and the linearity of cortisone in saliva was in the range of $0.2-40 \mathrm{ng} /$ $\mathrm{mL}$. The mean correlation coefficients of the two analytes were found to be $\geq 0.99$. The limit of detections (LOD) in serum and saliva were $100 \mathrm{pg} / \mathrm{mL}$ and $50 \mathrm{pg} / \mathrm{mL}$ for cortisol and cortisone, respectively. The precision and accuracy of cortisol and cortisone in serum and saliva samples are summarized in Table 1 and Table 2. Intra- and inter-batch accuracy and precision were determined at 4 different QC (LLOQ, LQC, MQC, and HQC) concentration levels. The linearity results demonstrated that the developed method is sensitive 
A

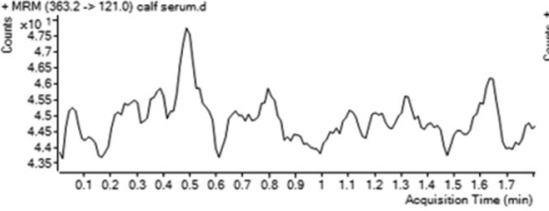

B

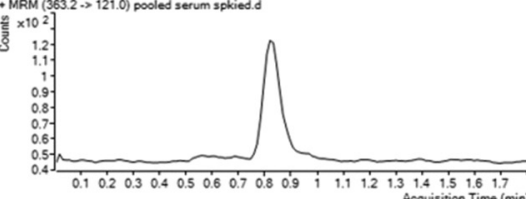

C
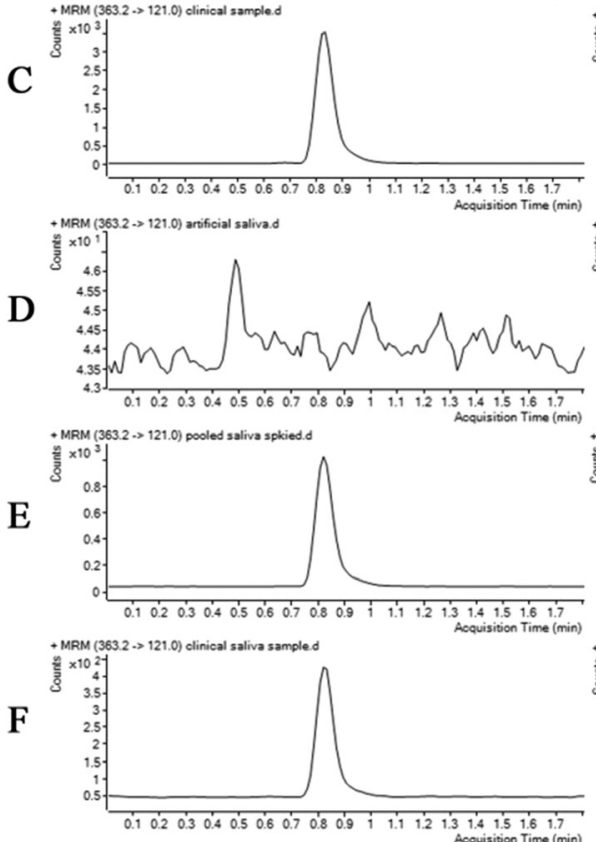
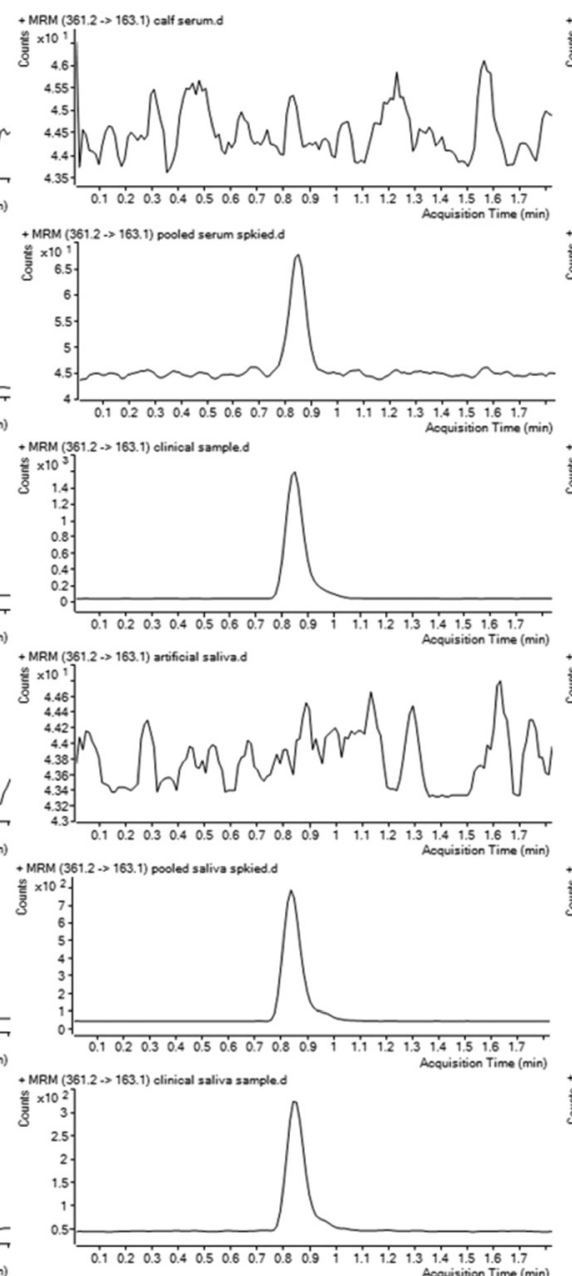
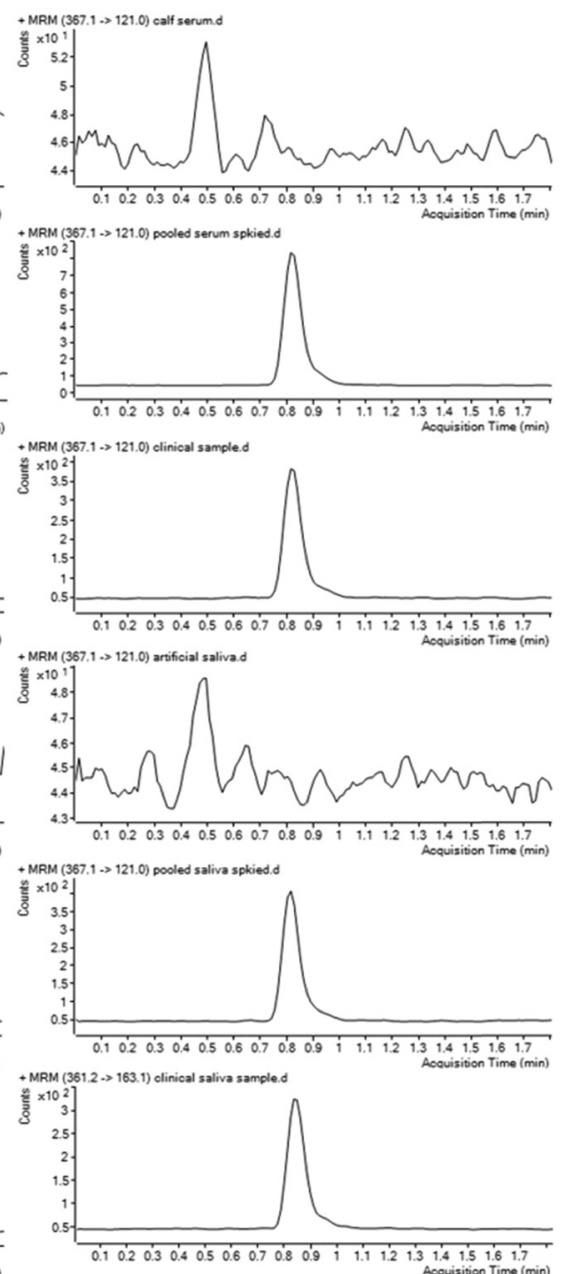

Figure 2. Chromatograms of cortisol (left panel), cortisone (middle panel), and cortisol-d4 (right panel) in (A) calf serum, (B) pooled human serum spiked with cortisol, cortisone, and cortisol-d4, (C) clinical serum sample, (D) artificial saliva, (E) pooled human saliva spiked with cortisol, cortisone, and cortisol-d4, and (F) clinical saliva sample

Table 1. Intra- and inter-assay precision and accuracy of serum and saliva cortisol

\begin{tabular}{|c|c|c|c|c|c|c|c|c|}
\hline & \multicolumn{4}{|c|}{ QC of serum } & \multicolumn{4}{|c|}{ QC of saliva } \\
\hline & LLOQ & LQC & MQC & HQC & LLOQ & LQC & MQC & HQC \\
\hline Spiked level (ng/mL) & 2 & 6 & 40 & 80 & 0.1 & 0.2 & 8 & 16 \\
\hline Mean matrix $(n=6)$ & $0^{a}$ & $0^{a}$ & $112.19^{b}$ & $112.19^{b}$ & $0^{a}$ & $0^{a}$ & $0.82^{b}$ & $0.82^{b}$ \\
\hline Intra-run mean $(n=6)$ & $1.82 \pm 0.10$ & $5.84 \pm 0.28$ & $148.81 \pm 3.05$ & $189.76 \pm 3.13$ & $0.100 \pm 0.008$ & $0.19 \pm 0.03$ & $9.03 \pm 0.20$ & $17.03 \pm 0.17$ \\
\hline Intra-run precision $(\%)$ & 5.37 & 4.81 & 1.92 & 1.57 & 3.96 & 8.60 & 2.18 & 1.01 \\
\hline Intra-run accuracy $(\%)$ & 91.16 & 96.45 & 98.02 & 98.88 & 100.02 & 97.46 & 102.43 & 101.27 \\
\hline Inter-day mean $(n=6)$ & $1.80 \pm 0.12$ & $5.80 \pm 0.25$ & $150.73 \pm 3.96$ & $189.99 \pm 2.31$ & $0.106 \pm 0.020$ & $0.20 \pm 0.03$ & $0.19 \pm 0.23$ & $16.97 \pm 0.19$ \\
\hline Inter-day precision $(\%)$ & 6.46 & 4.35 & 2.47 & 1.15 & 9.70 & 6.90 & 2.52 & 1.14 \\
\hline Inter-day accuracy $(\%)$ & 91.73 & 96.66 & 99.20 & 98.99 & 103.04 & 99.27 & 104.23 & 100.89 \\
\hline
\end{tabular}

${ }^{a}$ Cortisol- and cortisone-free matrices were used in the sample preparation.

${ }^{b}$ Pooled matrix was used in the sample preparation.

Table 2. Intra- and inter-assay precision and accuracy of serum and saliva cortisone

\begin{tabular}{|c|c|c|c|c|c|c|c|c|}
\hline & \multicolumn{4}{|c|}{ QC of serum } & \multicolumn{4}{|c|}{ QC of saliva } \\
\hline & LLOQ & LQC & MQC & HQC & LLOQ & LQC & MQC & HQC \\
\hline Spiked level (ng/mL) & 1 & 3 & 20 & 40 & 0.2 & 0.4 & 14 & 28 \\
\hline Mean matrix $(n=6)$ & $0^{a}$ & $0^{a}$ & $22.29^{b}$ & $22.29^{b}$ & $0^{a}$ & $0^{a}$ & $7.53^{b}$ & $7.53^{b}$ \\
\hline Intra-run mean $(n=6)$ & $1.16 \pm 0.04$ & $3.43 \pm 0.04$ & $42.42 \pm 0.86$ & $63.71 \pm 1.09$ & $0.18 \pm 0.01$ & $0.38 \pm 0.01$ & $21.10 \pm 0.13$ & $35.60 \pm 0.12$ \\
\hline Intra-run precision $(\%)$ & 3.70 & 1.28 & 2.02 & 1.71 & 10.44 & 2.06 & 1.20 & 0.75 \\
\hline Intra-run accuracy $(\%)$ & 116.46 & 114.34 & 100.30 & 102.27 & 95.43 & 91.93 & 96.31 & 100.47 \\
\hline Inter-day mean $(n=6)$ & $1.16 \pm 0.06$ & $3.37 \pm 0.18$ & $42.90 \pm 0.88$ & $63.40 \pm 0.86$ & $0.18 \pm 0.01$ & $0.39 \pm 0.01$ & $21.27 \pm 0.28$ & $35.45 \pm 0.27$ \\
\hline Inter-day accuracy $(\%)$ & 115.69 & 112.18 & 101.66 & 101.78 & 91.46 & 92.91 & 98.44 & 99.47 \\
\hline
\end{tabular}

enough to quantitatively determine the cortisol and cortisone in serum and saliva. The results of the developed method were precise and accurate, indicating that all values met the requirement of FDA guidelines. 
Table 3. Extract recovery and matrix effect for cortisol and cortisone in serum and saliva

\begin{tabular}{lccccc}
\hline & & $\begin{array}{c}\text { Spiked } \\
\text { level } \\
(\mathrm{ng} / \mathrm{mL})\end{array}$ & $\begin{array}{c}\text { Mean } \\
\text { matrix } \\
(n=6)\end{array}$ & $\begin{array}{c}\text { Extract recovery } \\
(\text { Mean } \pm \mathrm{SD}, \%)\end{array}$ & $\begin{array}{c}\text { Matrix } \\
\text { effect }(\%)\end{array}$ \\
\hline Serum & LQC & 6 & $0^{a}$ & $97.75 \pm 0.30$ & $93.82 \pm 6.82$ \\
cortisol & MQC & 40 & $112.19^{b}$ & $98.27 \pm 1.46$ & $98.87 \pm 6.57$ \\
& HQC & 80 & $112.19^{b}$ & $94.18 \pm 2.25$ & $106.39 \pm 3.32$ \\
Serum & LQC & 3 & $0^{a}$ & $98.93 \pm 3.01$ & $98.67 \pm 4.14$ \\
cortisone & MQC & 20 & $22.29^{b}$ & $95.32 \pm 2.48$ & $92.58 \pm 6.14$ \\
& HQC & 40 & $22.29^{b}$ & $93.06 \pm 1.69$ & $104.48 \pm 2.02$ \\
Saliva & LQC & 0.2 & $0^{a}$ & $89.95 \pm 6.76$ & $95.49 \pm 5.20$ \\
cortisol & MQC & 8 & $0.82^{b}$ & $94.24 \pm 1.78$ & $103.69 \pm 6.27$ \\
& HQC & 16 & $0.82^{b}$ & $90.51 \pm 1.33$ & $98.97 \pm 3.89$ \\
Saliva & LQC & 0.4 & $0^{a}$ & $96.71 \pm 1.23$ & $98.62 \pm 5.47$ \\
cortisone & MQC & 14 & $7.53^{b}$ & $90.22 \pm 4.03$ & $99.17 \pm 6.19$ \\
& HQC & 28 & $7.53^{b}$ & $97.94 \pm 3.29$ & $96.71 \pm 5.11$
\end{tabular}

${ }^{a}$ Cortisol- and cortisone-free matrices were used in the sample preparation.

${ }^{b}$ Pooled matrix was used in the sample preparation.

Extraction Recovery and Matrix Effects. The mean recoveries and matrix effects of the two analytes in serum and saliva are shown in Table 3. The recoveries for cortisol and cortisone were measured at 3 QC levels. The data suggested that the SLE extraction method provided reproducible recovery for human serum and saliva with acceptable variability. The results of the matrix effect experiment were in the range of $92.58-104.48 \%$, indicating that the matrix effects were acceptable in the serum and saliva samples. The co-eluting substances especially the endogenous did not interfere with the ionization of all the analytes.

Stability Studies. The stability experimental results are presented in Table 4 and Table 5. The stability results of cortisol and cortisone in the serum and saliva showed that all samples remained stable under all tested conditions with \%RSD < $9.68 \%$. The results showed that cortisol and cortisone were stable after 3 freeze-thaw cycles in serum and saliva. The stability results also illustrated that cortisol and cortisone were stable in human serum and saliva at room temperature for $24 \mathrm{~h}$ without protection from light and were stable in ultralow temperature refrigeration for 4 weeks.

Carryover and Dilution Integrity. No carry-over effects were observed after the highest calibration standard was injected. The precisions for dilution integrity were between 1.28 and $6.74 \%$, while the accuracy was within $1.37-8.19 \%$

Table 4. Stability results for cortisol in serum and saliva $(n=6)$

\begin{tabular}{|c|c|c|c|c|c|c|}
\hline Stability & $\begin{array}{l}\text { Spiked level } \\
(\mathrm{ng} / \mathrm{mL})\end{array}$ & $\begin{array}{c}\text { Mean concentration of } \\
\text { comparison samples }(\text { mean } \pm \mathrm{SD})\end{array}$ & $\begin{array}{l}\text { CV } \\
(\%)\end{array}$ & $\begin{array}{c}\text { Mean concentration of } \\
\text { comparison samples }(\text { mean } \pm \mathrm{SD})\end{array}$ & $\begin{array}{l}\mathrm{CV} \\
(\%)\end{array}$ & $\begin{array}{l}\text { Stability } \\
(\%)\end{array}$ \\
\hline Long-term & $6^{a}$ & $6.12 \pm 0.06$ & 7.98 & $5.48 \pm 0.06$ & 6.48 & 89.61 \\
\hline stability in & $80^{b}$ & $176.36 \pm 3.12$ & 4.37 & $175.49 \pm 2.59$ & 4.11 & 99.51 \\
\hline serum & $0^{c}$ & $92.99 \pm 8.08$ & 5.12 & $91.17 \pm 2.65$ & 2.88 & 98.04 \\
\hline Short-term & $6^{a}$ & $6.15 \pm 0.06$ & 8.81 & $5.85 \pm 0.05$ & 4.31 & 95.12 \\
\hline stability in & $80^{b}$ & $173.36 \pm 4.51$ & 4.63 & $173.06 \pm 3.43$ & 6.35 & 99.82 \\
\hline serum & $0^{c}$ & $107.58 \pm 5.16$ & 4.31 & $105.68 \pm 2.55$ & 5.69 & 98.23 \\
\hline \multirow{3}{*}{$\begin{array}{l}\text { Freeze-thaw } \\
\text { in serum }\end{array}$} & $6^{a}$ & $6.10 \pm 0.05$ & 8.01 & $5.70 \pm 0.04$ & 8.55 & 93.44 \\
\hline & $80^{b}$ & $175.44 \pm 3.38$ & 5.33 & $174.02 \pm 2.81$ & 4.89 & 99.19 \\
\hline & $0^{c}$ & $80.47 \pm 7.66$ & 5.39 & $77.35 \pm 2.37$ & 3.97 & 96.12 \\
\hline Long-term & $0.2^{a}$ & $0.19 \pm 0.03$ & 5.29 & $0.20 \pm 0.03$ & 6.09 & 102.56 \\
\hline stability in & $16^{b}$ & $16.82 \pm 4.18$ & 3.67 & $16.38 \pm 7.36$ & 7.66 & 97.37 \\
\hline saliva & $0^{c}$ & $1.42 \pm 0.06$ & 4.29 & $1.42 \pm 0.08$ & 6.06 & 99.72 \\
\hline Short-term & $0.2^{a}$ & $0.20 \pm 0.02$ & 3.98 & $0.19 \pm 0.06$ & 4.77 & 97.58 \\
\hline stability in & $16^{b}$ & $16.77 \pm 3.75$ & 3.39 & $16.88 \pm 5.42$ & 6.21 & 100.66 \\
\hline saliva & $0^{c}$ & $1.90 \pm 0.06$ & 3.21 & $1.88 \pm 0.04$ & 5.83 & 98.95 \\
\hline \multirow{3}{*}{$\begin{array}{l}\text { Freeze-thaw } \\
\text { in saliva }\end{array}$} & $0.2^{a}$ & $0.18 \pm 0.02$ & 4.19 & $0.18 \pm 0.05$ & 7.47 & 95.18 \\
\hline & $16^{b}$ & $15.92 \pm 3.33$ & 4.66 & $15.48 \pm 5.82$ & 3.63 & 97.24 \\
\hline & $0^{c}$ & $1.58 \pm 0.06$ & 6.29 & $1.55 \pm 0.07$ & 5.77 & 98.10 \\
\hline
\end{tabular}

${ }^{a}$ Cortisol- and cortisone-free matrices were used in the sample preparation.

${ }^{b}$ Pooled matrix was used in the sample preparation.

${ }^{c}$ Practical samples were used in the stability experiments.

Table 5. Stability results for cortisone in serum and saliva $(n=6)$

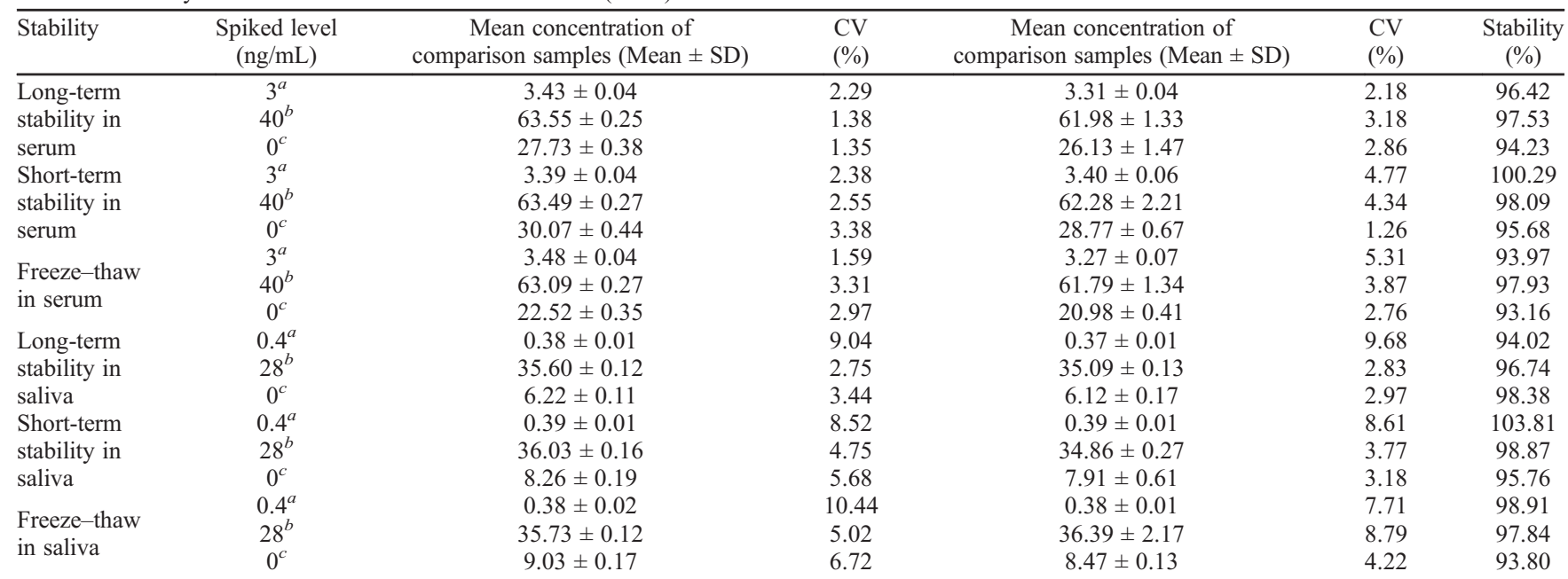

${ }^{a}$ Cortisol- and cortisone-free matrices were used in the sample preparation.

${ }^{b}$ Pooled matrix was used in the sample preparation.

${ }^{c}$ Practical samples were used in the stability experiments. 
for cortisol and $1.69-9.53 \%$ for cortisone. The acceptable inaccuracy and imprecision of the diluted serum and saliva samples were within $10 \%$.

\section{Correlation of Serum and Saliva Cortisol and Cortisone.} Both serum and saliva paired samples were determined in the 38 enrolled subjects. The mean $\pm \mathrm{SD}$ of cortisol and cortisone in serum were $111.27 \pm 47.25 \mathrm{ng} / \mathrm{mL}$ and $21.16 \pm 11.99 \mathrm{ng} / \mathrm{mL}$, and the mean $\pm \mathrm{SD}$ of cortisol and cortisone in saliva were $6.08 \pm 4.63 \mathrm{ng} / \mathrm{mL}$ and $9.23 \pm 6.46 \mathrm{ng} / \mathrm{mL}$, respectively. The correlations are presented in Figure 3. Serum cortisol was correlated with salivary cortisol $(r=0.443, P<0.001)$, and serum cortisone was correlated with salivary cortisone $(r=0.384, P<0.001)$. Modest correlations were found in both cortisol and cortisone. Some studies have found
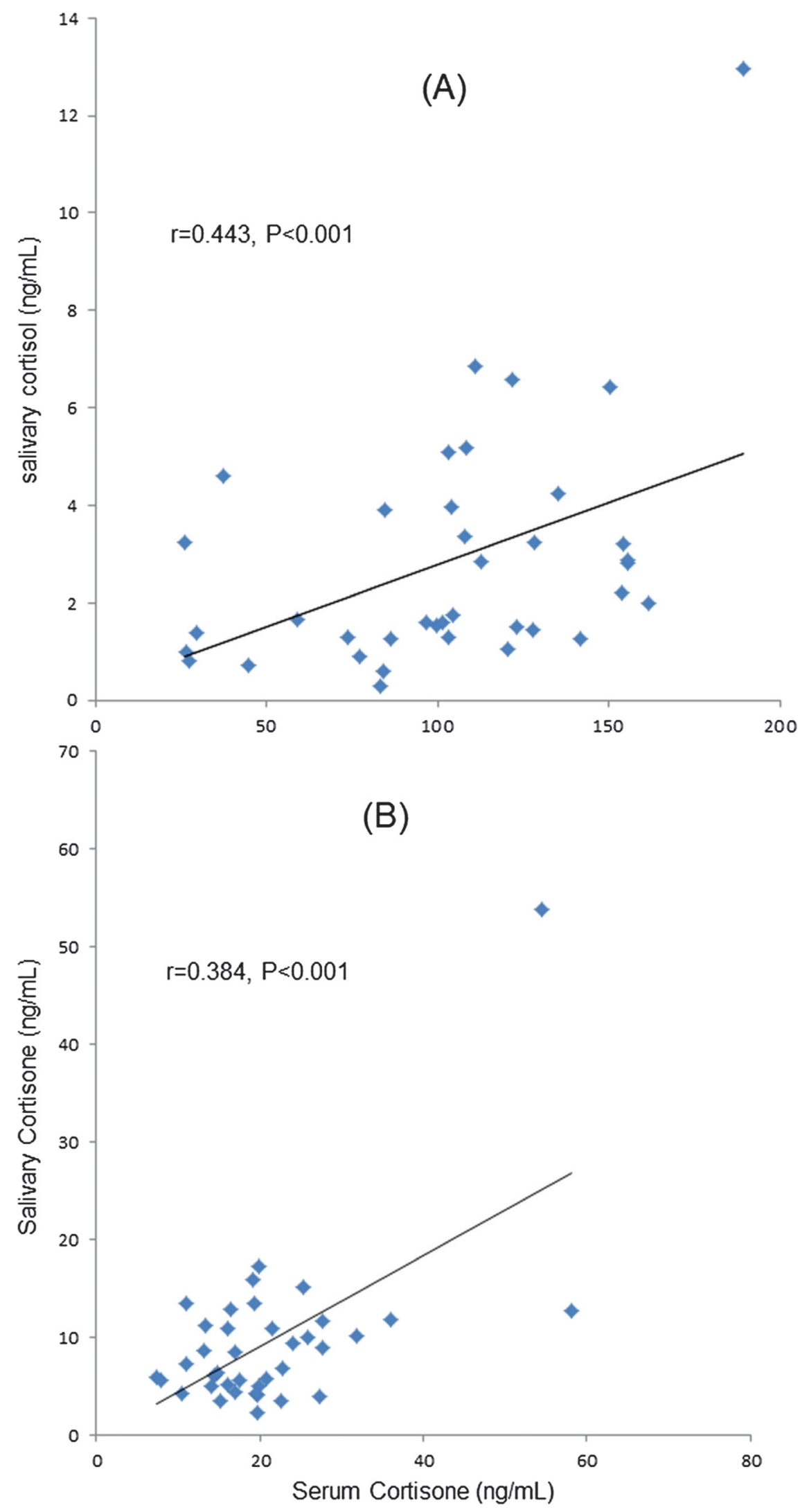

Figure 3. Relationship between serum and saliva cortisol (A) and cortisone (B) levels 
significant correlations between serum and salivary cortisol $[12,22]$; however, there is still some controversy and discussion regarding this issue. Some studies suggest that salivary cortisone reflects serum cortisol [23], but there was no correlation found in the present study. The correlation between cortisol and cortisone values in serum and saliva observed in the present study suggests that salivary values may be used as possible markers of cortisol and cortisone in the serum, further studies with a larger number of obtained samples are required to get more reliable results.

\section{Conclusion}

In conclusion, a fast and robust HPLC-MS/MS method for the determination of cortisol and cortisone from both serum and saliva by supported liquid extraction was validated. The LLOQ is more than adequate for utilization in clinical studies. Our study indicates that this HPLC-MS/MS procedure is reproducible and provides consistent quantification of cortisol and cortisone in serum and saliva. The superior recovery of the SLE method is an advantageous characteristic for clinical study. The extraction method showed the advantages of high speed, good efficiency, and easy automation. The bioanalytical method has successfully been used in the serum and saliva samples in patients with depressive disorders. The chromatographic run time of $2.0 \mathrm{~min}$ meets the requirements of high-throughput bioanalysis of cortisol and cortisone. The correlation results of serums and saliva suggest that salivary values can be used as markers of cortisol and cortisone in the blood. Salivary sampling could be a reliable and noninvasive alternative to measure hypothalamus-pituitary-adrenal axis states in clinical practice.

Acknowledgement. This work was supported by the Xiamen Municipal Bureau of Science and Technology Planning Project under grant no. 3502Z20164048, Natural Science Foundation of Fujian Province under grant no. 2019D016, Training Program for the Middle-Age and Young Key Talents by Health Commission of Fujian Province under grant no. 2018-ZQN-94, Educational Research Program for Middle-Age and Young Teachers of the Educational Committee of Fujian Province under grant no. JAT170698, and Innovation and Entrepreneurship Training Program for
College Students of Xiamen Medical College under grant no. 201712631004.

\section{References}

1. Blair, J.; Adaway, J.; Keevil, B.; Ross, R. Curr. Opin. Endocrinol., Diabetes Obes. 2017, 24, 161.

2. Zankert, S.; Bellingrath, S.; Wust, S.; Kudielka, B. M. Psychoneuroendocrinology 2019, 105, 86.

3. Lewitzka, U.; Bauer, M.; Ripke, B.; Bronisch, T.; Günther, L. Neuropsychobiology 2017, 75, 162.

4. O'Connor, D. B.; Green, J. A.; Ferguson, E.; O'Carroll, R. E.; O'Connor, R. C. Psychoneuroendocrinology 2017, 75, 183.

5. Papadopoulou, A.; Douzenis, A.; Christodoulou, C.; Gournellis, R.; Papageorgiou, C.; Markianos, M. Neuropsychobiology 2017, 76, 161.

6. Islam, M. R.; Islam, M. R.; Ahmed, I.; Moktadir, A. A.; Nahar, Z.; Islam, M. S.; Shahid, S. F. B.; Islam, S. N.; Islam, M. S.; Hasnat, A. SAGE Open Med. 2018, 6, 2050312118773953 . Doi: 10.1177/2050312118773953.

7. Williams, S. G.; Turner-Henson, A.; Davis, S.; Soistmann, H. C. Biol. Res. Nurs. 2017, 19, 65 .

8. Isaksson, J.; Hogberg, U.; Valladares, E.; Lindblad, F. Psychiatry Res. 2016, 240,376 .

9. Peng, R.; Li, Y. Compr. Psychiatry 2017, 76, 113-118. Doi: 10.1016/j. comppsych.2017.03.011. 308 .

10. El-Farhan, N.; Rees, D. A.; Evans, C. Ann. Clin. Biochem. 2017, 54,

11. Vieira, J. G. H.; Nakamura, O. H.; Carvalho, V. M. Arq. Bras. Endocrinol. Metabol. 2014, 58, 844 .

12. Montsko, G.; Tarjanyi, Z.; Mezosi, E.; Kovacs, G. L. Anal. Bioanal. Chem. 2014, 406, 2333.

13. Fragala, M. S.; Goldman, S. M.; Goldman, M. M.; Bi, C.; Colletti, J. D.; Arent, S. M.; Walker, A. J.; Clarke, N. J. J. Strength Cond. Res. 2018, 32,2425 .

14. Huayllas, M. K. P.; Netzel, B. C.; Singh, R. J.; Kater, C. E. Lab Med. 2018, 49, 259

15. Kosicka, K.; Siemiatkowska, A.; Szpera-Gozdziewicz, A.; Krzyscin, M.; Breborowicz, G.; Glowka, F. Ann. Clin. Biochem. 2018, 4563218783789.

16. Abujaber, F.; Corps Ricardo, A. I.; Rios, A.; Guzman Bernardo, F. J.; Rodriguez Martin-Doimeadios, R. C. J. Pharm. Biomed. Anal. 2019, 165, 141. 17. Peti, A. P. F.; Locachevic, G. A.; Prado, M. K. B.; de Moraes, L. A. B.; Faccioli, L. H. J. Mass Spectrom. 2018, 53, 423.

18. Tamashima, E.; Kai, T.; Todoroki, K.; Kawami, Y.; Itoyama, M.; Hayama, T.; Yoshida, H.; Yamaguchi, M.; Nohta, H. Bunseki Kagaku 2013, 62,719

19. Sauve, E. N.; Langodegard, M.; Ekeberg, D.; Oiestad, A. M. J. Chromatogr. B: Anal. Technol. Biomed. Life Sci. 2012, 883-884, 177.

20. Gangu Naidu, C.; Nageswara Rao, R.; Prasada Rao, A. V.; Nagesh Kumar, K.; Padiya, R.; Madhusudhan Rao, V. J. Chromatogr. Sci. 2018, 56, 879.

21. Ding, X.; Li, F.; McKnight, J.; Schmidt, C.; Strooisma, K.; Shimizu, H.; Faber, K.; Ware, J. A.; Dean, B. J. Pharm. Biomed. Anal. 2014, 100, 150.

22. Turpeinen, U.; Hämäläinen, E. Best Pract. Res., Clin. Endocrinol. Metab. 2013, 27, 795.

23 Debono, M.; Harrison, R. F.; Whitaker, M. J.; Eckland, D.; Arlt, W.; Keevil, B. G.; Ross, J. C. J. Clin. Endocrinol. Metab. 2016, 101, 1469. 